\title{
ANASTOMOSE ENTRE A ARTÉRIA CARÓTIDA EXTERNA E A ARTÉRIA CARÓTIDA INTERNA SUPRACLINÓIDEA UTILIZANDO ENXERTO DE VEIA SAFENA PARA TRATAMENTO DE ANEURISMA GIGANTE DO SEGMENTO CAVERNOSO DA CARÓTIDA INTERNA
}

\section{Relato de caso}

\author{
Hildo Azevedo Filho', Carolina Martins², Antonio Carvalho ${ }^{3}$, Geraldo Sá2, \\ Giovanni Grassi², Claudiana Cardoso'2, Gilberto Vilaça², Cícero Rodrigues ${ }^{4}$
}

\begin{abstract}
RESUMO - Procedimentos cirúrgicos alternativos para o tratamento de aneurismas "não clipáveis" da carótida intracavernosa incluem ligadura do vaso ou exclusão do segmento que origina a lesão. Essas técnicas estão associadas a riscos de complicações isquêmicas, mesmo em pacientes com sistema de colaterais funcionante. Portanto, um tratamento adequado requer revascularização encefálica para manter o fluxo sanguíneo nos territórios envolvidos. Relatamos o caso de uma paciente de 47 anos com sintomas e sinais isquêmicos embólicos e paralisia do III nervo craniano causados por aneurisma gigante, parcialmente trombosado, do segmento cavernoso da carótida interna. A paciente foi submetida a ponte anastomótica entre a carótida externa cervical e a carótida interna supraclinoidea utilizando enxerto de veia safena, seguido de exclusão do segmento vascular contendo o aneurisma, com bom resultado e sem complicações.
\end{abstract}

PALAVRAS -CHAVE:anastomose, carótida cavernosa, aneurisma intracraniano, aneurisma gigante, revascularização cerebral, veia safena.

\begin{abstract}
Saphenous vein graft bypass from the external carotid artery to the supraclinoid internal carotid artery to treat a giant aneurysm of the cavernous internal carotid: case report

ABSTRACT - Alternative surgical procedures to treat unclippable aneurysms of the intracavernous carotid artery include proximal vessel occlusion and trapping. Those techniques, even in patients with rich colateral vessels, are associated with risk of hemodynamic compromise and ischemic complications. Therefore, a safe treatment requires revascularization to maintain blood flow to the involved territories. We report the case of a 47-year-old female, with ischemic signs and symptoms and a right third nerve palsy caused by a giant aneurysm, partially trombosed, of the intracavernous carotid artery. The patient was submmited to trapping after a saphenous vein graft bypass from the external carotid artery to the supraclinoid internal carotid artery. The surgical result was good without complications.
\end{abstract}

KEY WORDS: bypass, cavernous carotid artery, giant intracranial aneurysm, cerebral revascularization, saphenous vein.

Os aneurismas do segmento cavernoso da artéria carótida interna representam 3 a $5 \%$ dos aneurismas intracranianos ${ }^{1}$ e correspondem a $14 \%$ daqueles originados da carótida interna². A história natural dessas lesões sugere que grande número delas permanecem assintomáticas e que a taxa de sangramento é menor do que aquelas situadas no compartimento subaracnóideo'. Portanto, a maioria desses aneurismas não requer tratamento². A intervenção está indicada quando ocorre ruptura aguda, compressão de nervos cranianos, episódios tromboembólicos ou ainda quando um aneurisma assintomático se estende para

\footnotetext{
Serviço de Neurocirurgia do Hospital da Restauração, Recife-PE: ${ }^{1}$ Professor Adjunto-Doutor em Neurocirurgia pela Universidade Federal de Pernambuco, PE; ${ }^{2}$ Neurocirurgião do Hospital da Restauração; ${ }^{3}$ Neurorradiologista do Hospital da Restauração; ${ }^{4}$ Cirurgião Vascular do Hospital da Restauração.
}

Recebido 4 Janeiro 1999, recebido na forma final 9 Outubro 2000. Aceito 18 Outubro 2000. 
o espaço subaracnóideo ${ }^{2}$, oferecendo risco considerável de sangramento.

O tratamento ideal para qualquer tipo de aneurisma consiste em excluí-lo da circulação sanguínea, sem alterar o fluxo no vaso que the deu origem ou em seus ramos ${ }^{2}$. A clipagem direta é a modalidade de tratamento que mais se aproxima desse objetivo. No entanto, a exposição microcirúrgica de aneurismas do segmento cavernoso da carótida consiste em grande desafio, considerando as relações anatômicas da carótida com os nervos que trafegam no seio cavernoso e a possibilidade de sangramento. Mesmo quando uma exposição adequada é obtida, características como o tamanho da lesão, formato do colo, presença de calcificações ou placas de ateroma e outras limitações técnico/cirúrgicas podem tornar esses aneurismas inadequados para a clipagem $^{2}$. Procedimentos alternativos incluem embolização com balões ou "coils", microcirurgia reconstrutiva vascular, ligadura da carótida com ou sem revascularização encefálica ou uma combinação dessas técnicas. Para Sundt Jr' ${ }^{1}$ a ligadura e ponte anastomótica para os aneurismas da carótida cavernosa são a segunda melhor solução para o reparo dessas lesões.

Relatamos o caso de uma paciente com sintomas e sinais compressivos e tromboembólicos decorrentes de aneurisma da carótida intracavernosa, em que o tratamento escolhido foi a exclusão do segmento que originava a lesão, após a construção de ponte anastomótica de alto débito.

\section{CASO}

MJS, 47 anos, feminina, natural de Palmares, Zona da Mata de Pernambuco, foi admitida no Serviço de Neurocirurgia do Hospital da Restauração - Recife em abril de 1998, com história de cefaléia têmporo-orbitária associada a desvio lateral progressivo do olho direito, há cerca de 1 ano. Poucas horas antes da admissão hospitalar a paciente foi despertada do sono por cefaléia de forte intensidade e mesma distribuição, que rapidamente se tornou holocraniana, associada a paresia e parestesias no dimídio esquerdo, além de ptose palpebral direita.

Exame físico. Na admissão foi constatada síndrome piramidal desproporcionada, de predomínio faciobraquial à esquerda e déficit completo do III nervo à direita. Durante o internamento hospitalar foi constatada a presença de hipertensão arterial, até então desconhecida pela paciente, instituindo-se terapêutica dietética e medicamentosa.

Exames de imagem. A tomografia computadorizada (TC) mostrou lesão hiperdensa parasselar à direita medindo $1,8 \times 2,0 \mathrm{~cm}$ nos seus maiores diâmetros, associada a erosão da parede do seio esfenoidal e área hipodensa

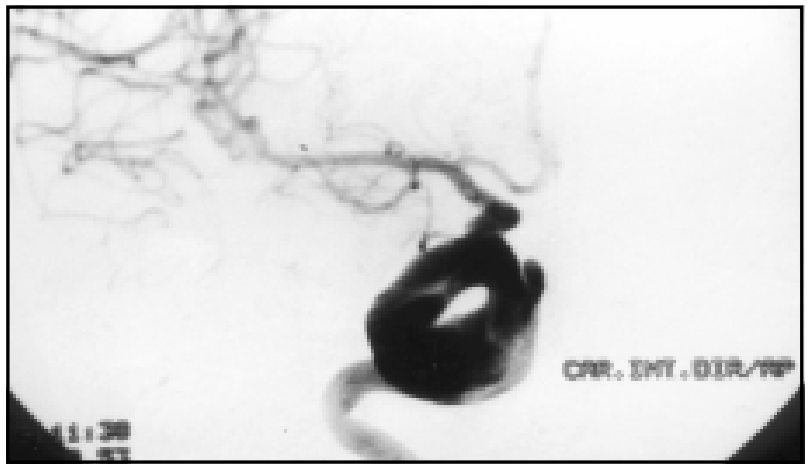

Fig 1. Angiografia pré-operatória da carótida direita em AP: grande aneurisma parcialmente trombosado do segmento cavernoso da carótida.

fronto-têmporo-insular direita que determinava apagamento parcial do corno frontal do ventrículo lateral direito. Havia ainda assimetria entre os lobos frontais, sendo o polo frontal esquerdo reduzido em volume. $O$ estudo angiográfico demonstrou que a lesão hiperdensa parasselar visibilizada à TC correspondia a um aneurisma gigante, parcialmente trombosado, do segmento cavernoso da carótida interna direita (Fig 1), associado a área hipovascular no óperculo frontal e ínsula direitos, com sinais de oclusão embólica dos ramos em candelabro da artéria cerebral média deste lado. $O$ teste de compressão da carótida direita revelou enchimento lento através da carótida esquerda com retardo venoso de cerca de 3 segundos. A artéria comunicante posterior direita estava pérvia, havendo opacificação da carótida supraclinóide e ramos parietais silvianos quando a injeção de contraste foi realizada na vertebral direita, dominante.

Procedimento cirúrgico. A paciente foi submetida a safenectomia esquerda e exposição das carótidas cervicais à direita. Em seguida foi realizada craniotomia frontotêmporo-orbitária direita com remoção extradural da clinóide anterior e dissecção microcirúrgica de todo o vale silviano, com exposição completa da carótida incluindo a emergência da artéria oftálmica direita. $\mathrm{O}$ aneurisma pode ser visibilizado, ocupando e distorcendo o seio cavernoso direito. Cinco mil unidades de heparina foram administradas por via intravenosa nesse momento. A construção da ponte anastomótica entre a carótida externa e a carótida interna supraclinóidea se iniciou com a anastomose na região cervical seguindo-se a tunelização do enxerto por sob o zigoma (Fig 2). Pulsações fortes, semeIhantes à carótida, foram verificadas após o enchimento do enxerto. A anastomose intracraniana término-lateral com a carótida supraclinoidea foi realizada após clipagem provisória da carótida interna, distal à oftálmica, assim como da comunicante posterior direita, visto haver fluxo por esta última, colateral como demonstrado na angiografia. O tempo total de construção da ponte anastomótica foi de três horas. Ao final da construção da anastomose intracraniana foram retirados os clips da comunicante posterior, carótida interna e finalmente o clip 


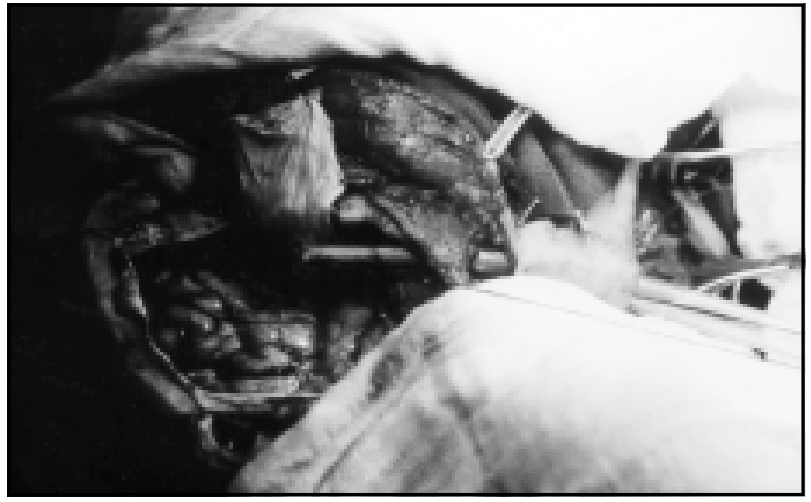

Fig. 2. Tunelização do enxerto de safena, após a anastomose cervical, por sob o arco zigomático, utilizando tubo de toracostomia.

provisório do enxerto, observando-se presença de fluxo e pulsações. Foi feita então a ligadura da carótida interna cervical proximal à emergência da artéria oftálmica direita.

Pós-operatório. A paciente foi mantida em uso de heparina de baixo peso molecular e antiagregantes plaquetários, sendo readmitida na enfermaria já no segundo dia do pós operatório.

A angiografia de controle, no oitavo dia de pós operatório ( $8^{\circ}$ DPO ) demonstrou a presença de fluxo sangüíneo entre o tronco da carótida externa e a porção supraclinóidea da carótida interna direitas, determinando enchimento anterógrado satisfatório dos ramos da cerebral anterior e média deste lado, além de oclusão da carótida interna desde a porção cervical até a porção supraclinóidea, onde foi possível identificar a presença de clip metálico (Fig 3).

A paciente recebeu alta no $18^{\circ} \mathrm{DPO}$. Acompanhamento ambulatorial no quarto mês do pós operatório mostrou redução da espasticidade no membro parético, sem sinais de regressão da oftalmoparesia.

\section{DISCUSSÃO}

No passado, a ligadura da carótida interna representou o tratamento de escolha dos aneurismas da porção cavernosa da carótida interna. A elevada taxa de complicações isquêmicas, 35 a 59\%, tanto precoces como tardias, associada ao desenvolvimento das técnicas microcirúrgicas levaram ao surgimento de outras abordagens ${ }^{3}$. As complicações isquêmicas eram decorrentes da perda do fluxo sangüíneo pela carótida ligada sem que existisse um sistema de colaterais adequado ${ }^{1}$ ou resultava de êmbolos desgarrados do coto vascular trombosado ("stump emboli")4. A revascularização cerebral realizada previamente à ligadura do vaso foi idealizada como uma forma de manter o fluxo sangüíneo para os territórios ameaçados, protegendo o paciente de complicações isquêmicas ${ }^{2}$.

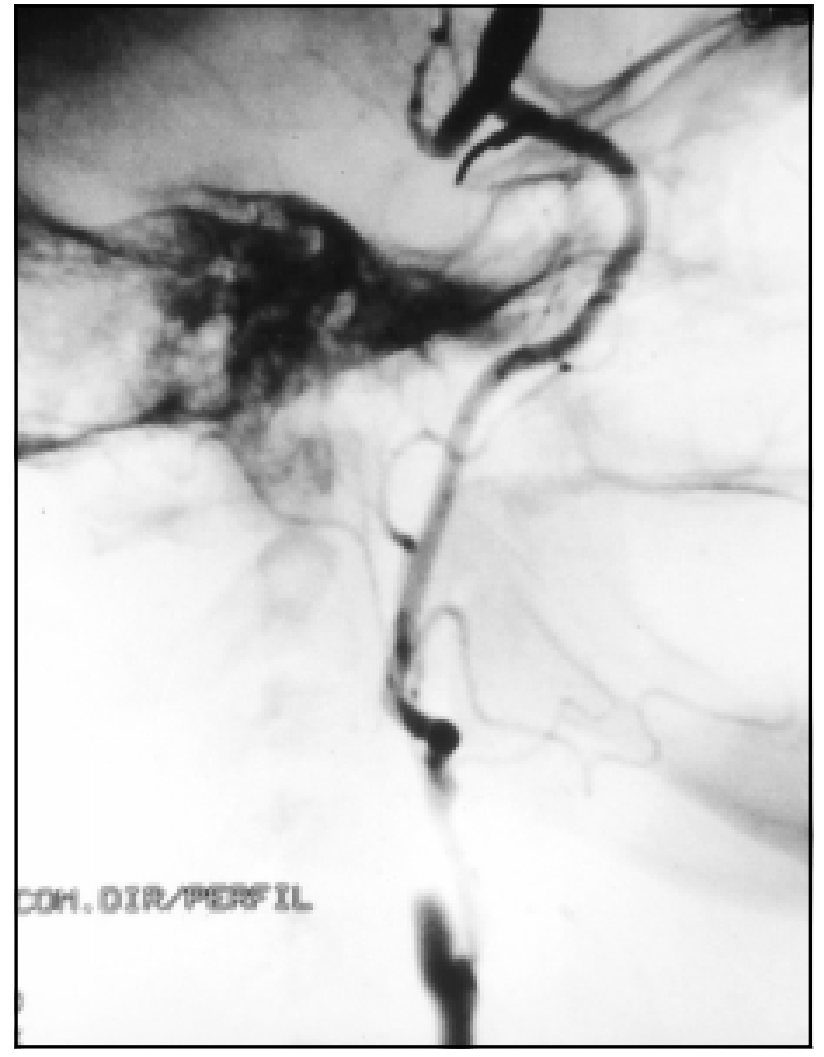

Fig. 3. Angiografia pós-operatória da carótida direita em perfil: patência do enxerto $\mathrm{CE}-\mathrm{Cl}$, com enchimento dos vasos distais. Oclusão da carótida interna desde a porção suprabulbar até a porção supraclinóidea ( clip metálico ), com exclusão do aneurisma.

A era das anastomoses microvasculares surgiu com o "bypass" da carótida externa/carótida interna, ACE- ACl, na forma dos shunts artéria-temporalsuperficial-artéria-cerebral-média , ATS-ACM, como proposto por Yasargil na década de $70^{3,5}$. Essas pontes anastomóticas, consideradas, mais tarde, de baixo fluxo, além de apresentarem predisposição à oclusão por trombose pelo fluxo lento ${ }^{6}$, não eram capazes de suprir o território vascular envolvido $2,3,4,6$, mais uma vez resultando em morbidade e mortalidade relacionada a eventos isquêmicos.

Isso impulsionou o desenvolvimento das técnicas de enxertos longos e anastomoses proximais. $O$ enxerto utilizando a veia safena entre a circulação extracraniana e intracraniana foi realizado inicialmente para promover fluxo colateral de alto volume ${ }^{1}$ em pacientes com doença cérebro-oclusiva avançada ${ }^{6}$, mais tarde se estendendo como alternativa viável na revascularização cerebral para o tratamento de aneurismas gigantes ou das lesões neoplásicas da base do crânio que requeriam a oclusão da carótida interna ${ }^{5,6}$.

Esse tipo de anastomose, assim como os enxertos venosos utilizados em cirurgia cardiovascular, se 
correlaciona com maior índice de falha durante o primeiro ano após a cirurgia, $43 \%$ dessas falhas ocorrendo nas primeiras 24 horas. No entanto, ao contrário dos enxertos periféricos e cardíacos, a taxa de oclusão do enxerto utilizado entre a circulação extra e intracraniana permanece constante entre 1 e 1,5\% por ano, alcançando taxas de patência cumulativa de $86 \%$ em um ano e $73 \%$, treze anos após a cirurgia ${ }^{6}$.

Para minimizar a oclusão precoce do enxerto, grande atenção deve ser dada ao detalhe técnico durante a construção das anastomoses, associado ao uso judicioso de anticoagulação, infusão de fluidos e controle pressórico ${ }^{4,6}$. É importante que exista um bom fluxo no enxerto venoso após a anastomose proximal. A qualidade do pulso se relaciona diretamente com a construção da anastomose proximal. Se o pulso na veia não se assemelha ao da carótida, o enxerto deve ser retirado e a anastomose refeita ${ }^{1}$.

Segundo Regli e colaboradores ${ }^{6}$, os maiores índices de falência do enxerto venoso a longo prazo são verificados quando utilizam-se veias de diâmetro menor que 6 milímetros (38\% de falhas), quando a anastomose proximal é realizada com a artéria subclávia $(50 \%)$ ou quando endarterectomia carotídea concomitante foi utilizada ( $67 \%$ de falhas).

Por outro lado, quando as anastomoses proximais envolveram a carótida, ou ainda, quando as veias foram anastomosadas a grandes troncos intracranianos, especialmente a carótida interna supraclinoidea como realizado em nossa paciente - as menores taxas de falência a longo prazo foram observadas ${ }^{6}$.

A utilização da carótida externa cervical para a anastomose proximal ao invés da carótida petrosa (bypass $\mathrm{C}_{3}-\mathrm{C}_{5}$ ) como proposto pelo grupo de Speltzler' ${ }^{2}$, é considerada mais simples. A anatomia dessa área, familiar tanto para neurocirurgiões quanto cirurgiões vasculares, permitiu a criação de equipe multidisciplinar, aliando as técnicas microcirúrgicas à experiência em construção de anastomoses. Isso pode ser especialmente importante em serviços com pequena experiência em cirurgias de revascularização cerebral.
A verificação da patência do enxerto no pósoperatório é usualmente feita através da angiografia digital ${ }^{7}$. Nos últimos anos, técnicas não invasivas têm sido aprimoradas com esse propósito. A angioressonância tem demonstrado boa correlação com a angiografia digital no estudo da patência dos enxertos ${ }^{7}$. Além de dispensar o uso de contraste, esse método ( especialmente quando consideradas as técnicas "phase-contrast e time-of-flight" ) é capaz de mostrar todos os vasos dentro do volume escolhido, analisando a contribuição do suprimento extracraniano ao fluxo cerebral sob condições fisiológicas ${ }^{7,8}$.

A verificação clínica da patência do enxerto através da avaliação do pulso na região pré auricular é preconizada por alguns autores ${ }^{6}$. A tunelização da safena por sob o zigoma, como realizado por nós, dificulta tal avaliação; porém protege o enxerto de traumatismos e de compressão extrínseca.

\section{REFERÊNCIAS}

1. Sundt TM Jr. Saphenous vein bypass for aneurysms of the intracavernous portion of the internal carotid artery. In Wilson CB (ed). Neurosurgical procedures: personal approachs to classic operations, Baltimore: Williams \& Wilkins, 1992:96-118.

2. Vishteh AG, Sankla, SK, Lawton, MT, Spetzler, RF. Surgical management of intracavernous artery aneurysms:BNI experience. BNI Quart, 1997;13:4-13.

3. Al-Mefty O, Origitano TC. Aneurysms of the cavernous sinus: treatment options and considerations. In Youmans JR (ed ). Neurological surgery, Philadelphia:Saunders,1996:1320-1334.

4. Hacein-Bey L, Connolly S Jr, Duong H, et al.. Treatment of inoperable carotid aneurysms with endovascular carotid oclusion after extracranial-intracranial bypass surgery. Neurosurgery, 1997;41:1225-1234.

5. Meneses MS, Ramina R, Jackowski AP, Pedrozo AA, Pacheco RB, Tsubouchi MH. Middle cerebral artery revascularization: anatomical studies and considerations on the anastomosis site. Arq Neuropsiquiatr 1997; 55:16-24

6. Regli L, Piepgras DG, Hansen C. Late patency of long saphenous vein bypass grafts to the anterior and posterior cerebral circulation. J Neurosurg 1995;83: 806-811.

7. Praharaj SS, Coulthard A, Gholkar A, English P, Mendelow AD. Magnetic resonance angiographic assessment after extracranial-intracranial bypass surgery. J Neurol Neurosurg Psychiatry 1996;60:439-441.

8. Kodoma T, Suzuki Y, Yano K, Watanabe K, Ueda T, Asada K. Phasecontrast MRA in the evaluation of EC-IC bypass patency. Clin Radiol 1995;50:459-465.

9. Yeh H, Tomsick T. Obliteration of a giant carotid aneurysm after extracranial-to-intracranial bypass surgery: case report. Surg Neurol 1997;48:473-476. 